Nonlinear models in macroeconometrics

Timo Teräsvirta

CREATES Research Paper 2017-32 


\title{
Nonlinear models in macroeconometrics
}

\author{
Timo Teräsvirta \\ CREATES, Aarhus University \\ C.A.S.E., Humboldt-Universität zu Berlin
}

September 12, 2017

\begin{abstract}
This article contains a short review of nonlinear models that are applied to modelling macroeconomic time series. Brief descriptions of relevant models, both univariate, dynamic single-equation, and vector autoregressive ones are presented. Their application is illuminated by a number of selected examples.

Keywords: Markov-switching model, nonlinear time series, random coefficient model, smooth transition model, threshold autoregressive model, vector autoregressive model

JEL Classification Codes: C32; C51; E00

Acknowledgement: This research has been partly supported by the Center for Research in Econometric Analysis of Time Series (CREATES), funded by the Danish National Research Foundation, Grant No. DNRF 78.
\end{abstract}

This article has been written for the Oxford Research Encyclopedia in Economics and Finance. 


\section{Introduction}

The number of nonlinear models in the macroeconometric literature is large. A restriction imposed for this article is that only parametric time series models are considered. This excludes, among other things, nonlinear crosssectional models and nonparametric time series models. Nonlinear panel models constitute an exception because they bear rather strong resemblance to some of the nonlinear models discussed in this article. Deterministic models (with random noise) that have occasionally been applied to macroeconomic time series are not reviewed either. Linear models with breaks may also viewed as nonlinear, but the huge literature of structural breaks is omitted from consideration here.

The focus will be on models that do not contain many theory restrictions on their structure. Univariate models naturally belong to this category, and in the vector case, nonlinear vector autoregressive models will receive most of the attention. Further, all models to be highlighted are conditional mean models. Although models of the conditional variance have also been applied to macroeconomic time series, see for example the seminal contribution by Engle (1982), they are much more popular in financial applications and are left outside this work.

Since applications of nonlinear vector models to macroeconomic time series have become common later than applications of their univariate counterparts, the former models will receive more attention than the latter. Presentations of nonlinear vector models are accompanied by relevant macroeconomic examples, so the reader can see where and how the models can and have so far been applied.

The plan of the article is as follows. Univariate models are considered in Section 2 and dynamic single-equation ones in Section 3. Multivariate (vector) models are the topic of Section 4. Two nonlinear panel models are briefly presented in Section 5. Section 6 contains final remarks and suggestions for further reading.

\section{Univariate autoregressive and single-equation regression models}

\subsection{Switching regression and smooth transition models}

Models with more than one regime have a long history in statistics, time series analysis and econometrics. The first models were regression models with independent observations. Quandt (1958) considered a switching regression 
model in which the regression equation, including the error term, switches according to a random switch variable. As an example Quandt used a consumption equation in which a switch in the regime occurs when the interest rate exceeds a certain value. Let $y_{i}$ be the consumption, $x_{i}$ the income and $j_{i}$ the interest rate. The model is

$$
y_{i}=a+b x_{i}+\varepsilon_{1 i}
$$

for $j_{i} \geq \bar{j}$, and

$$
y_{i}=c+d x_{i}+\varepsilon_{2 i}
$$

otherwise, where $(a, b) \neq(c, d)$. The independent errors $\varepsilon_{k i} \sim \mathcal{N}\left(0, \sigma_{k i}^{2}\right)$, $k=1,2, \sigma_{1 i}^{2} \neq \sigma_{2 i}^{2}$. The author suggested that the parameters of the model be estimated by maximising the log-likelihood using a grid over the values of $j_{i}$. (In this discussion, however, he used time as the switch variable.) Due to discontinuity of the log-likelihood, this is by and large how parameter estimation is carried out in all switching regression models. Testing the hypothesis that there is only a single regime was also considered. Quandt (1958) acknowledged the fact that the true switch-point, if any, is unknown, but time was not yet ripe for a rigorous analysis of this testing problem.

Bacon and Watts (1971) argued that instead of having an abrupt shift from one regime to the other, one could make the transition smooth. Using previous notation, their smooth transition model has the form

$$
y_{i}=a+b x_{i}+\left(c+d x_{i}\right) G\left(x_{i}\right)+\varepsilon_{i}
$$

where the transition function $G\left(x_{i}\right)$ is a bounded continuous function, monotonic in $x_{i}$. The authors used the two-parameter hyperbolic tangent function that is bounded between zero and one but pointed out that many other functions would be equally possible. They assumed the error variance to be constant for all values of $G\left(x_{i}\right)$ and adopted a Bayesian approach to estimating the parameters of the model. The application in Bacon and Watts (1971) was not an economic one but had to do with a chemical.

Interestingly, in the econometrics literature, Goldfeld and Quandt (1972, pp. 263-264) independently presented the smooth transition regression model as a solution to the estimation problem in the switching regression model (1) and (2) with switch variable $j_{i}$. The idea was to approximate the switch by a smooth continuous function to make the log-likelihood well behaved and maximise the likelihood using standard nonlinear optimisation algorithms.

These models were adapted to time series a few years later. The breakthrough in the univariate case, called the threshold autoregressive (TAR) model, came with the paper by Tong and Lim (1980). The model has the 
form

$$
y_{t}=\sum_{j=1}^{r}\left(\phi_{j}^{\prime} \mathbf{w}_{t}+\varepsilon_{j t}\right) I\left(c_{j-1}<y_{t-d} \leq c_{j}\right)
$$

where $I(A)$ is the indicator function: $I(A)=1$ when $A$ is true, and zero otherwise, $c_{0}, c_{1}, \ldots, c_{r}$ are threshold parameters, $c_{0}=-\infty, c_{r}=\infty$, and $\mathbf{w}_{t}=\left(1, y_{t-1}, \ldots, y_{t-p}\right)^{\prime}$. If $r=1$, the model is linear. Furthermore, $\phi_{j}=$ $\left(\phi_{0 j}, \phi_{1 j}, \ldots, \phi_{p j}\right)^{\prime}$ such that $\phi_{i} \neq \phi_{j}$ for $i \neq j$, and $\varepsilon_{j t}=\sigma_{j} \varepsilon_{t}$ with $\left\{\varepsilon_{t}\right\} \sim$ $\operatorname{iid}(0,1)$, and $\sigma_{j}>0, j=1, \ldots, r$. The TAR model is called self-exciting when the threshold variable is a lag of $y_{t}$ as in (4). In many economic applications the TAR model is assumed to have two regimes:

$$
y_{t}=\left(\phi_{1}^{\prime} \mathbf{w}_{t}+\varepsilon_{1 t}\right) I\left(s_{t} \leq c_{1}\right)+\left(\phi_{2}^{\prime} \mathbf{w}_{t}+\varepsilon_{2 t}\right)\left\{1-I\left(s_{t} \leq c_{1}\right)\right\} .
$$

A comprehensive account of the model and its statistical properties can be found in Tong (1990).

The autoregressive counterpart of the smooth transition model (3) was introduced by Chan and Tong (1986). The smooth transition autoregressive (STAR) model is

$$
y_{t}=\boldsymbol{\phi}_{1}^{\prime} \mathbf{w}_{t}+\boldsymbol{\phi}_{2}^{\prime} \mathbf{w}_{t} G\left(y_{t-d}\right)+\varepsilon_{t}
$$

where, following previous notation, $\varepsilon_{j t}=\sigma \varepsilon_{t}$ with $\left\{\varepsilon_{t}\right\} \sim \operatorname{iid}(0,1)$, and $\sigma>0$. The transition function $G\left(y_{t-d}\right)$ of Chan and Tong (1986) is the cumulative distribution function of the standard normal variable. The logistic function introduced by Maddala (1977, p. 396) has become the most popular choice in the literature. Teräsvirta (1994) suggested pairing the logistic function

$$
G_{L}\left(y_{t-d}\right)=\left(1+\exp \left\{-\gamma\left(y_{t-d}-c\right)\right\}\right)^{-1}, \gamma>0
$$

with the exponential function

$$
G_{E}\left(y_{t-d}\right)=1-\exp \left\{-\gamma\left(y_{t-d}-c\right)^{2}\right\}, \gamma>0
$$

previously used in a slightly more restricted form in the exponential autoregressive (EAR) model by Haggan and Ozaki (1981). When $\gamma \rightarrow \infty$ in (7), the model converges to a two-regime TAR model, whereas the EAR model or the more general ESTAR model (6) with (8) becomes linear. Teräsvirta (1994) discussed the choice between the logistic and the exponential transition function and STAR model specification more generally as well. Jansen and Teräsvirta (1996) suggested another variant of the logistic function that is close to the exponential transition function but contains one parameter more than the latter. A STAR model with this transition function converges to a special case of a TAR model with three regimes when $\gamma \rightarrow \infty$. The 
LSTAR model may be generalised to a multiple-transition model as in van Dijk and Franses (1999), but many economic applications rely on a singletransition model.

A few macroeconomic time series such as interest rates and unemployment rate are rather persistent. Many authors tend to model them as random walks without drift. Lanne and Saikkonen (2002) provided a useful nonlinear alternative that mimics the behaviour of these series but can be stationary. Their model is a TAR model in which only the intercept is switching:

$$
y_{t}=\sum_{j=1}^{r} \phi_{0 j} I\left(c_{j-1}<y_{t-d} \leq c_{j}\right)+\phi^{\prime} \mathbf{w}_{t}+\varepsilon_{t}
$$

where $\boldsymbol{\phi}=\left(\phi_{1}, \ldots, \phi_{p}\right)^{\prime}$ and $\mathbf{w}_{t}=\left(y_{t-1}, \ldots, y_{t-p}\right)^{\prime}$. Given that the intercept remains bounded, this model has the same stationarity conditions as the linear AR model. The authors fitted their model to two monthly time series: the Swiss Franc Euro exchange rate and a UK Treasury bill rate.

The argument in the indicator function in (5) may be replaced by an unobservable discrete stochastic variable $s_{t}$ that obtains $r$ different values $s_{t} \in\{1, \ldots, r\}$, say, and has a (typically first-order) Markov structure. The transition (or staying if $i=j$ ) probabilities

$$
p_{i j}=\operatorname{Pr}\left\{s_{t}=j \mid s_{t-1}=i\right\}, i, j=1, . ., r .
$$

determine the probability for the process to switch from regime $i$ to regime $j$ at time $t$ and are parameters to be estimated. The resulting model is the Markov switching or hidden Markov autoregressive (MSAR) model. It may be written as follows:

$$
y_{t}=\sum_{j=1}^{r} \boldsymbol{\phi}_{j}^{\prime} \mathbf{w}_{t} I\left(s_{t}=j\right)+\varepsilon_{t}
$$

with $\phi_{i} \neq \phi_{j}$ for $i \neq j$, where $\varepsilon_{j t}=\sigma \varepsilon_{t}$ with $\left\{\varepsilon_{t}\right\} \sim \operatorname{iid} \mathcal{N}(0,1)$. Douc, Moulines and Rydén (2004) considered this model and proved consistency and asymptotic normality for the maximum likelihood estimators of its parameters. Maximum likelihood estimation of the MS regression model was already studied by Lindgren (1978).

Hamilton (1989) introduced a different MSAR model. The latent variable has the same values and transition probabilities as (11) with (10) but a different structure:

$$
\begin{aligned}
y_{t} & =\mu\left(s_{t}\right)+\sum_{i=1}^{p} \phi_{i}\left\{y_{t-i}-\mu\left(s_{t-i}\right)\right\}+\varepsilon_{t} \\
& =\left\{\mu\left(s_{t}\right)-\sum_{i=1}^{p} \phi_{i} \mu\left(s_{t-i}\right)\right\}+\sum_{i=1}^{p} \phi_{i} y_{t-i}+\varepsilon_{t} .
\end{aligned}
$$


where $\mu(i) \neq \mu(j)$ for all $i \neq j$. From (12) it is seen that the model bears similarity to (9) in that only the intercept is switching. If $r=2$, as is often the case in macroeconomic applications, the latent switching intercept can obtain $2^{p+1}$ different values.

\subsection{Time-varying parameter models and Bayesian tech- niques}

Another nonlinear model may be constructed from a linear AR model by making its coefficients random. An early example is the autoregressive model

$$
y_{t}=\phi_{1 t}^{\prime} \mathbf{w}_{t}+\varepsilon_{t}
$$

where $\phi_{1 t}=\left(\phi_{1 t}, \ldots, \phi_{p t}\right)^{\prime}$ and $\varepsilon_{t} \sim \operatorname{iid} \mathcal{N}\left(0, \sigma^{2}\right)$, see Andel (1976) and Nicholls and Quinn (1982). The time-varying elements $\phi_{i t} \sim \operatorname{iid}\left(\phi_{i}, \sigma_{i}^{2}\right)$. The survey by Swamy and Tavlas (1995) concentrates on random coefficient regression models and contains a number of macroeconomic examples. In many macroeconomic applications, the random coefficients are instead made persistent by assuming that they follow a random walk:

$$
\phi_{i t}=\phi_{i t-1}+\lambda_{i t} \nu_{i t}
$$

where $\lambda_{i t} \geq 0, i=1, \ldots, p$. Furthermore, $\nu_{i t} \sim \operatorname{iid} \mathcal{N}\left(0, \sigma^{2}\right), i=1, \ldots, p$, and independent of $\varepsilon_{t}$. When $\lambda_{i t}=0$ for all $i$ and $t$, (13) is a linear autoregressive model. In order to prevent $\left\{y_{t}\right\}$ from exploding during the sample period, the parameters $\lambda_{i t}$ have to be small. Koop and Potter (2001) showed how TVP-AR models are estimated using suitably chosen prior distributions and numerical techniques. Their Bayesian approach also allows model comparisons using Bayes factors.

Bayesian methods also enable simultaneous comparisons of large numbers of time series models. Koop and Potter (2000) discuss building nonlinear models with these methods and apply them to two macroeconomic series, the growth rate of the quarterly real US GDP, 1954(1)-1987(4), and the annual British industrial production index, 1700-1992. They define 11 classes of models, linear models, linear models with one or two structural breaks, linear models with one or two outliers and two- and three regime TAR models. In addition, they specify each model with a different number of lags and with homoskedastic or heteroscedastic errors. The idea is to compute the posterior model probability for every model and find out the most probable model or models. In the case of the US GDP, this approach favours a model with a single structural break and heteroskedastic errors, whereas a three-regime TAR model with homoskedastic errors has the highest posterior probability 
for the industrial production index. Since publication of Koop and Potter (2000), increasing computational power has no doubt increased the attraction of model comparisons of this kind.

\subsection{Other applications}

Univariate STAR and TAR models have been applied to a large number of macroeconomic variables, including industrial production, unemployment and inflation, to name perhaps the most important ones. The main reason for nonlinearity has been asymmetry. For example, dynamic behaviour of the growth rate of industrial production has in many countries been different during recessions and expansions. Early examples of this can be found in Teräsvirta and Anderson (1992) who fitted STAR models to quarterly series of growth rates of industrial production in various countries. Similarly, the MSAR model has been applied to characterizing business cycles, in which case the latent variable represents the phase of the cycle. See for example Montgomery, Zarnowitz, Tsay and Tiao (1998) for (11) with (10) and Hamilton (1989) for (12). In macroeconomic applications, the number of regimes in MSAR models is typically chosen a priori and not determined from the data.

\section{Dynamic single-equation regression models}

Augmenting a univariate nonlinear autoregressive model by exogenous variables leads to a dynamic nonlinear regression model. To give an example, the smooth transition regression (STR) model is obtained as

$$
y_{t}=\phi_{1}^{\prime} \mathbf{w}_{t}+\phi_{2}^{\prime} \mathbf{w}_{t} G\left(s_{t}\right)+\boldsymbol{\psi}_{1}^{\prime} \mathbf{x}_{t}+\boldsymbol{\psi}_{2}^{\prime} \mathbf{x}_{t} G\left(s_{t}\right)+\varepsilon_{t}
$$

where $\boldsymbol{\psi}_{1}^{\prime} \neq \boldsymbol{\psi}_{2}^{\prime}, \mathbf{x}_{t}=\left(x_{1 t}, \ldots, x_{k t}\right)^{\prime}$ is a vector of at least weakly exogenous variables, $G\left(s_{t}\right)$ is (for example) a logistic transition function, and $s_{t}$ is a stationary transition variable. Possibilities include $s_{t}=x_{j t}$ and $s_{t}=y_{t-d}$, but $s_{t}$ may also be an exogenous variable not in $\mathbf{x}_{t}$. STR models have been applied to modelling money demand in Germany and the UK. For an application to the long annual UK money demand series, originally considered and modelled by Ericsson, Hendry and Prestwich (1998), see Teräsvirta and Eliasson (2001). A comprehensive model building strategy for STR models is discussed in Teräsvirta (1998). 


\section{Vector nonlinear models}

Univariate time series models, linear and nonlinear, can be used for forecasting, but describing relationships between macroeconomic variables requires multivariate models, unless exogeneity assumptions can be made. Many of the models fitted to macroeconomic time series are general time series models that have found application in a wide range of areas. They typically nest a standard linear vector autoregressive (VAR) model and are generalisations of corresponding univariate models. A few frequently applied nonlinear VAR models will be discussed below.

\subsection{Disequilibrium models}

Before considering VAR models, however, the focus will be on a family of models arising from economic theory propositions. There exist situations in economics in which markets do not clear, that is, the ex ante demand and supply quantities cannot be equated, i.e., the market is in disequilibrium. For example, a disequilibrium in labour markets may be due to wages that do not adjust downwards. Government-controlled apartment rents are another example of this type of disequilibrium. Further, credit rationing may create a market in disequilibrium: there may be excess demand because the banks may not lend money to firms that they consider too risky recipients of loans.

Fair and Jaffee (1972) were the first to define a general disequilibrium model. It contains both a demand and a supply equation. Borrowing the notation in Teräsvirta et al. (2010, Chapter 2), the demand equation equals

$$
D_{t}=\boldsymbol{\alpha}_{0}^{\prime} \mathbf{x}_{t}^{D}+\alpha_{1} p_{t}+\varepsilon_{t}^{D}
$$

where $D_{t}$ is the quantity demanded at time $t, \mathbf{x}_{t}^{D}$ is the vector of variables, except the price, affecting the demand, $p_{t}$ is the price at time $t, \alpha_{1}<0$ (the price has a negative effect on demand) and $\varepsilon_{t}^{D}$ is an error term. The supply equation is

$$
S_{t}=\boldsymbol{\beta}_{0}^{\prime} \mathbf{x}_{t}^{S}+\beta_{1} p_{t}+\varepsilon_{t}^{S}
$$

where $S_{t}$ is the quantity supplied at time $t, \mathbf{x}_{t}^{S}$ is the vector of variables, other than the price, affecting the supply, $\beta_{1}>0$ (the price has a positive effect on supply) and $\varepsilon_{t}^{S}$ is an error term. When $D_{t} \neq S_{t}$, only the smaller one of the two quantities is observed. This is indicated by completing (14) and (15) by the 'min-condition' for the observed quantity:

$$
D_{t}^{\text {obs }}=\min \left(D_{t}, S_{t}\right)
$$

The resulting system defined by (14), (15) and (16) is strongly nonlinear. It does not nest a linear system. 
Fair and Jaffee (1972) applied the model to the demand and supply of housing starts in the US. The number of housing starts is a nonstationary variable, and both the demand and supply equation contain a time trend to reflect different nonstationarities in these two equations. Estimation is carried out by quantifying the difference between demand and supply as follows:

$$
D_{t}-S_{t}=\gamma \Delta p_{t}, \gamma>0 .
$$

This means that the min-condition is replaced by (17). This leads to a singleequation switching regression model

$$
\begin{aligned}
D_{t}^{\mathrm{obs}} & =\left(\boldsymbol{\alpha}_{0}^{\prime} \mathbf{x}_{t}^{D}+\alpha_{1} p_{t}-\frac{1}{\gamma} \Delta p_{t}+\varepsilon_{t}^{D}\right) I\left(\Delta p_{t} \geq 0\right) \\
& +\left(\boldsymbol{\beta}_{0}^{\prime} \mathbf{x}_{t}^{S}+\beta_{1} p_{t}-\frac{1}{\gamma} \Delta p_{t}+\varepsilon_{t}^{S}\right)\left\{1-I\left(\Delta p_{t} \geq 0\right)\right\}
\end{aligned}
$$

where the switch is instantaneous (observed at the same time as $p_{t}$ ) and the switch-point known. The error process switches as well. An increase in price (in the application a lagged mortgage interest rate) decreases demand for housing starts whereas a decrease increases the supply.

For maximum likelihood estimation of disequilibrium models with the min-condition, see Maddala and Nelson (1974). A survey of disequilibrium models can be found in Maddala (1983, Chapter 10). For a Bayesian approach and more discussion about the model, see Bauwens, Lubrano and Richard (1999, Section 8.6).

\subsection{Vector smooth transition regression model}

The first VAR model to be considered here is the Logistic Vector STR (LVSTR) model. It is a generalisation of the single-equation STR model to the vector case. Following Hubrich and Teräsvirta (2013), the LVSTR model of order $p$ may be defined as follows:

$$
\begin{aligned}
\mathbf{y}_{t}= & \boldsymbol{\mu}_{0}+\mathbf{G}\left(\boldsymbol{\gamma}, \mathbf{c} ; \mathbf{s}_{t}\right) \boldsymbol{\mu}_{1}+\sum_{j=1}^{p}\left\{\boldsymbol{\Phi}_{j}+\mathbf{G}\left(\boldsymbol{\gamma}, \mathbf{c} ; \mathbf{s}_{t}\right) \boldsymbol{\Psi}_{j}\right\} \mathbf{y}_{t-j} \\
& +\left(\boldsymbol{\Gamma}+\mathbf{G}\left(\boldsymbol{\gamma}, \mathbf{c} ; \mathbf{s}_{t}\right) \boldsymbol{\Xi}\right) \mathbf{x}_{t}+\boldsymbol{\varepsilon}_{t}
\end{aligned}
$$

where $\mathbf{y}_{t}$ is an $m \times 1$ vector of stationary variables, $\mathbf{x}_{t}$ is an $n \times 1$ vector of stationary exogenous variables, $\boldsymbol{\mu}_{0}$ and $\boldsymbol{\mu}_{1}$ are $m \times 1$ intercept vectors, $\boldsymbol{\Phi}_{j}$ and $\boldsymbol{\Psi}_{j}, j=1, \ldots, p$, are $m \times m$ parameter matrices, and $\boldsymbol{\Gamma}$ and $\boldsymbol{\Xi}$ are $m \times n$ parameter matrices. Each row of the composite matrix $\left[\boldsymbol{\mu}_{1}, \boldsymbol{\Psi}_{1}, \ldots, \boldsymbol{\Psi}_{p}, \boldsymbol{\Xi}\right]$ 
has to contain at least one nonzero element. The $m \times m$ transition matrix $\mathbf{G}\left(\boldsymbol{\gamma}, \mathbf{c} ; \mathbf{s}_{t}\right)$ has the following form:

$$
\mathbf{G}\left(\boldsymbol{\gamma}, \mathbf{c} ; \mathbf{s}_{t}\right)=\operatorname{diag}\left\{G_{1}\left(\gamma_{1}, \mathbf{c}_{1}, s_{1 t}\right), \ldots, G_{m}\left(\gamma_{m}, \mathbf{c}_{m}, s_{m t}\right)\right\}
$$

where $s_{i t}, i=1, \ldots, m$, are stationary transition variables. The error vector $\boldsymbol{\varepsilon}_{t} \sim \operatorname{iid}(\mathbf{0}, \boldsymbol{\Sigma})$ where $\boldsymbol{\Sigma}>0$. When $G_{j}\left(\gamma_{j}, \mathbf{c}_{j}, s_{j t}\right), j=1, \ldots, m$, are standard logistic functions,

$$
G_{j}\left(\gamma_{j}, \mathbf{c}_{j}, s_{j t}\right)=\left(1+\exp \left\{-\gamma_{j}\left(s_{j t}-c_{j}\right)\right\}\right)^{-1}, \gamma_{j}>0
$$

and $\boldsymbol{\Gamma}=\boldsymbol{\Xi}=\mathbf{0}$, the model (18) is stable if both $\left|\mathbf{I}_{m}-\sum_{j=1}^{p} \boldsymbol{\Phi}_{j} z^{j}\right| \neq 0$ and $\left|\mathbf{I}_{m}-\sum_{j=1}^{p}\left(\boldsymbol{\Phi}_{j}+\boldsymbol{\Psi}_{j}\right) z^{j}\right| \neq 0$ for $|z| \leq 1$.

A special case found in many applications is the one in which (19) is simplified to

$$
\mathbf{G}\left(\boldsymbol{\gamma}, \mathbf{c} ; \mathbf{s}_{t}\right)=G\left(\gamma, \mathbf{c} ; s_{t}\right) \mathbf{I}_{m}
$$

where a single transition function controls the shift in all equations. Camacho (2004) considered this model and devised a modelling strategy for it. Replacing the transition function in $(21)$ by $I\left(s_{t} \leq c\right)$ and setting $\boldsymbol{\Gamma}=\boldsymbol{\Xi}=\mathbf{0}$ in (18) yields the two-regime Vector Threshold Autoregressive (VTAR) model by Tsay (1998).

Neither LVSTR nor the VTAR model is identified when the data-generating process is linear. To avoid the estimation of unidentified models, it follows that linearity has to be tested before fitting either of the two models to the data.

Interaction between the real and financial sectors of the economy has become under scrutiny especially after the financial crisis of years 20072008. Schleer and Semmler (2015) apply the LVSTAR model $(\boldsymbol{\Gamma}=\boldsymbol{\Xi}=\mathbf{0}$ in (18)) to study this interaction in 11 euro area countries. They assume that there may be two extreme regimes: a low and a high (financial) stress regime. To study the effects of financial stress to the economy they construct for each country a two-dimensional LVSTAR model with the growth rate and the corresponding ZEW Financial Condition Index (FCI) for the euro area financial conditions as variables. The transition variable $s_{t}$ in $(21)$ is a lag of FCI of that country. Linearity is tested and rejected before specifying and estimating a nonlinear model. Generalised impulse response functions (GIRF), see for example Koop, Pesaran and Potter (1996) or Teräsvirta et al. (2010, Chapter 15), computed from the estimated model are used to interpret the results. They show that the response to a financial shock is stronger and longer-lasting during financial stress than when the stress is low.

Caggiano, Castelnuovo and Figueres (2017) consider the effect of policy uncertainty on central macroeconomic variables of the US economy during 
different phases of the business cycle. The policy uncertainty is measured by an index constructed by Baker, Bloom and Davis (2016). The variables are the six-term moving average of the monthly growth rate of industrial production, the unemployment rate, the year-on-year CPI inflation and the federal funds rate. In addition, the model contains a binary policy uncertainty dummy variable based on the uncertainty index. After testing and rejecting linearity, the authors construct an LVSTAR model for these variables. A smoothed and lagged growth rate of the industrial production functions as the transition variable in (21). Even here, GIRF are used to illustrate the results. They show that exogenous policy uncertainty shocks have stronger effects on the economy in recessions than in expansions. See also Caggiano, Castelnuovo and Groshenny (2014) for an application of the LVSTAR model to estimating the effects of policy uncertainty on the US unemployment rate.

\subsection{Vector smooth transition error correction model}

In the previous section it has been assumed that the variables in the model are stationary. Many macroeconomic variables are, however, nonstationary and some of them 'move together', in which case they may be assumed (or shown) to be linearly cointegrated. Based on this assumption, Gefang (2012) used a Vector Smooth Transition Error Correction (VSTEC) model to study the money-output relationship. An interesting thing is that she estimates the model using Bayesian techniques. Bayesian methods in general are frequently used in the estimation of nonlinear VAR models because they tend to alleviate numerical problems present in the estimation of some of them.

The Logistic VSTEC model in Gefang (2012) is obtained by reparameterising the LVSTAR model, (18) with $\boldsymbol{\Gamma}=\boldsymbol{\Xi}=\mathbf{0}$, and the transition function (21) as follows:

$$
\begin{aligned}
\Delta \mathbf{y}_{t}= & \mathbf{D}_{t} \boldsymbol{\nu}_{0}+G\left(\gamma, \mathbf{c} ; s_{t}\right) \mathbf{D}_{t} \boldsymbol{\nu}_{1}+\boldsymbol{\Pi}_{0} \mathbf{y}_{t-1}+G\left(\gamma, \mathbf{c} ; s_{t}\right) \boldsymbol{\Pi}_{1} \mathbf{y}_{t-1} \\
& +\sum_{j=1}^{p}\left\{\boldsymbol{\Phi}_{j}+G\left(\gamma, \mathbf{c} ; s_{t}\right) \boldsymbol{\Psi}_{j}\right\} \Delta \mathbf{y}_{t-j}+\boldsymbol{\varepsilon}_{t}, \gamma>0
\end{aligned}
$$

where $\Delta \mathbf{y}_{t}$ is assumed stationary in the mean, $\mathbf{D}_{t}$ contains the deterministic components (in (18) the intercept, in (22) the intercept and the linear time trend), and the $m \times m$ matrix $\boldsymbol{\Pi}_{i}=\mathbf{A}_{i} \mathbf{B}_{i}^{\prime}$, with $\operatorname{rank}\left(\mathbf{A}_{i}\right)=\operatorname{rank}\left(\mathbf{B}_{i}\right)=q_{i}<$ $m$ and $i=0,1$. Note that the ranks need not be equal. The cointegrating relationships defined in $\mathbf{B}_{i}$ are thus assumed to change with the regime, which would probably complicate the classical specification and estimation procedure quite substantially. Details of how this and other difficulties are handled in the Bayesian framework are discussed in the paper. It may be 
mentioned, however, that to avoid the aforementioned identification problem: (22) is not identified if the true data-generating process is linear, the prior distribution for the slope parameter $\gamma$ is bounded away from zero. There are 18 candidates for the transition variable $s_{t}$, and the Bayesian approach allows one to investigate all of them.

The model in the application is a four-variable LSTVAR consisting of the seasonally adjusted industrial production index, the seasonally adjusted M2 money stock, the producer price index for all commodities and the secondary market rate on three-month Treasury bills. The observations are monthly US data from 1959(1) to 2006(12). Bayesian posterior probabilities are calculated for 3138 models in total. The models with the highest posterior probability have one factor in common: they all suggest that money nonlinearly Granger causes output.

\subsection{Vector threshold autoregressive model}

As already mentioned, replacing the transition function in (21) by $I\left(s_{t} \leq c\right)$ and setting $\boldsymbol{\Gamma}=\boldsymbol{\Xi}=\mathbf{0}$ in (18) yields the two-regime Vector Threshold Autoregressive (VTAR) model by Tsay (1998). The author developed a useful strategy for building such models. The application in that paper is to financial series and is therefore not considered here. For an interesting macroeconomic application we turn to Galvão (2006) whose VTAR model has an extra twist: it also allows for a break in the series. The Vector Structural Break Threshold Autoregressive (VSBTAR) model is defined as follows:

$$
\begin{aligned}
\mathbf{y}_{t} & =\left[\left(\boldsymbol{\mu}^{(1)}+\sum_{j=1}^{p} \boldsymbol{\Phi}_{j}^{(1)} \mathbf{y}_{t-j}\right) I_{z}\left(s_{t-d} \leq r_{1}\right)+\left(\boldsymbol{\mu}^{(2)}+\sum_{j=1}^{p} \boldsymbol{\Phi}_{j}^{(2)} \mathbf{y}_{t-j}\right)\right. \\
& \left.\times\left\{1-I_{z}\left(s_{t-d} \leq r_{1}\right)\right\}\right] I_{t}\left(t \leq t_{0}\right) \\
& +\left[\left(\boldsymbol{\mu}^{(3)}+\sum_{j=1}^{p} \boldsymbol{\Phi}_{j}^{(3)} \mathbf{y}_{t-j}\right) I_{z}\left(s_{t-d} \leq r_{3}\right)+\left(\boldsymbol{\mu}^{(2)}+\sum_{j=1}^{p} \boldsymbol{\Phi}_{j}^{(2)} \mathbf{y}_{t-j}\right)\right. \\
& \left.\times\left\{1-I_{z}\left(s_{t-d} \leq r_{3}\right)\right\}\right]\left\{1-I_{t}\left(t \leq t_{0}\right)\right\}+\boldsymbol{\varepsilon}_{t}
\end{aligned}
$$

where $s_{t}$ is the threshold variable, $d>0, \varepsilon_{t} \sim \operatorname{iid}(\mathbf{0}, \boldsymbol{\Sigma}), r_{1}$ and $r_{3}$ are switchpoints and $t_{0}$ is a break-point. When $I_{t} \equiv 1,(23)$ collapses into a VTAR model, whereas when $I_{z} \equiv 1$, the model is a linear VAR with a break at $t=t_{0}$. The standard two-regime VTAR model thus becomes

$$
\begin{aligned}
\mathbf{y}_{t} & =\left(\boldsymbol{\mu}^{(1)}+\sum_{j=1}^{p} \boldsymbol{\Phi}_{j}^{(1)} \mathbf{y}_{t-j}\right) I\left(s_{t-d} \leq r_{1}\right)+\left(\boldsymbol{\mu}^{(2)}+\sum_{j=1}^{p} \boldsymbol{\Phi}_{j}^{(2)} \mathbf{y}_{t-j}\right) \\
& \times\left\{1-I\left(s_{t-d} \leq r_{1}\right)\right\}+\boldsymbol{\varepsilon}_{t} .
\end{aligned}
$$


The structure of (23) resembles that of the single-equation time-varying STAR model of Lundbergh, Teräsvirta and van Dijk (2003) with the extension that $r_{1} \neq r_{3}$. The model is aimed at describing the relationship between economic growth and interest rate spread. The purpose is to investigate the claim that the spread forecasts economic growth in recessions but not in expansions. The possibility that the relationship is changing over time is considered as well.

Specification of VSBTAR models requires care due to the double transition structure. Since the model is only identified under the alternative, Galvão (2006) applies supremum linearity tests. For these tests, see for instance Hansen (1996) or Teräsvirta et al. (2010, Section 5.5). The parameters are estimated by conditional least squares or by maximum likelihood conditionally on the switch-points $r_{1}, r_{3}$ and $t_{0}$. A three-dimensional grid is formed for these three parameters, and the (global) optimum of the objective function (log-likelihood or the sum of squared errors) yields the estimates for them.

The VSBTAR model is applied to predicting recessions in the US economy. There exists literature suggesting that interest rate spreads are useful in predicting output growth only when the growth rate is negative but not otherwise. This relationship could be described by a VTAR model. But then, Galvão (2006) also cites research suggesting that spread may have lost its predictive power, which could be investigated by a linear VAR model with breaks. Considering these proposals jointly leads to the bivariate VSBTAR model whose variable are the output growth and the spread between the long- and short-term interest rates. The observations are quarterly from $1953(2)$ to $2002(4)$. The model selection procedure supports the choice of the VSBTAR model.

It is not possible here to describe the forecasting procedure or how success in predicting recessions is measured. Galvão (2006) reports that the VSBTAR model performs better than its competitors, VAR and VTAR, insample, whereas the latter two models are 'more robust', meaning that they outperform the more complicated VSBTAR model out-of-sample. The results show that the estimate of the break-point in the VSBTAR model is changing when new data become available. This may not be surprising because the model allows exactly one break, and it may be reasonable to expect the most conspicuous shift in parameters to be the one determining the location of the sole break-point. 


\subsection{Vector threshold cointegration}

The LVSTEC model (22) was preceded by a vector threshold cointegration (VTC) model that Balke and Fomby (1997) introduced. The VTC model with three regimes can be written as follows:

$$
\Delta \mathbf{y}_{t}=\sum_{j=1}^{3}\left(\boldsymbol{\mu}_{j}+\boldsymbol{\alpha}_{j} \boldsymbol{\beta}^{\prime} \mathbf{y}_{t-1}+\sum_{k=1}^{p-1} \boldsymbol{\Psi}_{k}^{(j)} \Delta \mathbf{y}_{t-k}+\boldsymbol{\varepsilon}_{t}^{(j)}\right) I\left(c_{j-1}<s_{t-1} \leq c_{j}\right)
$$

for $p \geq 2$, where $\boldsymbol{\beta}$ and $\boldsymbol{\alpha}_{j}, j=1,2,3$, are $m \times 1$ vectors and $\left\{\boldsymbol{\varepsilon}_{t}^{(j)}\right\}$ is a sequence of independent but not identically distributed vectors with mean $\mathbf{0}$ and covariance matrix $\boldsymbol{\Sigma}_{j}$. In this model, the cointegrating relationship $s_{t}=\boldsymbol{\beta}^{\prime} \mathbf{y}_{t}$ and the switch variable equals $s_{t-1}$. If $c_{1}<0$ and $c_{2}>0$ (assuming as before that $c_{0}=-\infty$ and $c_{3}=+\infty$ ) and, furthermore, $\boldsymbol{\alpha}_{2}=\mathbf{0}$, the model describes a situation in which there is a band around the equilibrium $s_{t}=0$ such that within the band no adjustment towards the equilibrium takes place. When $p=1$, the lags of $\Delta \mathbf{y}_{t}$ vanish from the model. Saikkonen (2008) considered (25) assuming $\boldsymbol{\Psi}_{k}^{(j)}=\boldsymbol{\Psi}_{k}$ and $\boldsymbol{\varepsilon}_{t}^{(j)}=\boldsymbol{\varepsilon}_{t}, j=1,2,3 ; k=1, \ldots, p-1$. Furthermore, he also discussed the case in which instead of three distinct regimes the transition from one extreme to the other is smooth and described by two logistic transition functions.

The VTC model is often applied to describing the relationship between two interest rates. The argument is that transaction costs prevent the adjustment inside a band. In these cases often $\boldsymbol{\beta}=(1,-1)$. Besides, it may be assumed that the band is symmetric around zero: $c_{1}=-c_{2}$ in (25). Bec and Rahbek (2004) studied a pair of short-term and long-term German interest rates using a VTC model. Their univariate tests rejected the unit root hypothesis against a stationary threshold alternative for both series. The authors then fitted a univariate TAR model to $\boldsymbol{\beta}^{\prime} \mathbf{y}_{t}$ and, using a supremum linearity test, found that linearity was rejected. From this they concluded that the two series may be nonlinearly cointegrated and fit a VTC model to them.

Anderson (1997) suggested another nonlinear adjustment mechanism to consider the treasury bill market. The model is (25) except for two differences. First, the indicator function in (25) is replaced by the exponential transition function (8) with $c=0$ (as defined in the paper, $s_{t}=0$ is the equilibrium point at time $t)$. second, the delay $d=1$, and third, $\varepsilon_{t} \sim \operatorname{iid}(\mathbf{0}, \boldsymbol{\Sigma})$. This makes the adjustment smooth and nonlinear such that the drift towards the equilibrium first increases and becomes constant when $\left|s_{t-1}\right|$ becomes sufficiently large. The argument for this transition function is that different treasury bill owners face different transaction costs, in which case a sharp 
band is not a suitable description of the aggregate.

It may be mentioned that the (bivariate) vector threshold error correction model is substantially generalised by Cai, Gao and Tjøstheim (2017) who apply their model to characterising the relationship between the US federal funds rate controlled by the Federal Reserve and the three-month Treasury Bill rate. The results suggest that 'the Federal Reserve tends to adjust the federal funds rate as a response to the market interest rates.' A detailed treatment of the model is not possible here.

\subsection{Vector Markov Switching Autoregressive models}

Like the univariate smooth transition or threshold autoregressive models, the univariate Markov switching model can also be generalised to a vector model. The two-regime Vector Markov Switching Autoregressive (VMSAR) model may be obtained by replacing the indicator function in $(24)$ by $I\left(s_{t}=i\right)$ where $s_{t}$ is latent and $i=1,2$. The dynamic behaviour of the latent variable is defined as in the univariate case. For a review, see Krolzig (1997).

VMSAR models are quite popular in macroeconomics. As their univariate counterparts, they are suitable for situations in which it can be assumed (sometimes because of lack of information) that the probability of switching regimes is constant over time and does not depend on any observable indicator variable. Like the VTAR model, the VMSAR model nests a linear VAR model. It has the same property as the VTAR model: the VMSAR model is not identified when the true model is a linear VAR.

Warne and Vredin (2006) considered the question whether the unemployment is more (or less) volatile when inflation is high than when it is low. This is done for three countries, the US, the UK and Sweden. We choose the US to illustrate their work. The authors begin by constructing a theory model and continue by deriving its time series counterpart, a bivariate VAR model. Since there is a possibility that the two series are cointegrated, they first estimate a linear error correction VAR model. Misspecification tests for the model based on monthly US data from 1959(1) to 1998(12) show that the estimated model is not satisfactory: the errors are autocorrelated and seem to contain conditional heteroskedasticity.

The authors next consider a bivariate VMS error correction (VMS-EC) model for $\mathbf{y}_{t}=\left(y_{I N F, t}, y_{U, t}\right)^{\prime}$. It has the following form:

$$
\Delta \mathbf{y}_{t}=\sum_{j=1}^{2}\left(\boldsymbol{\mu}_{j}+\boldsymbol{\alpha}_{j} \boldsymbol{\beta}^{\prime} \mathbf{y}_{t-1}+\sum_{k=1}^{p-1} \boldsymbol{\Psi}_{k}^{(j)} \Delta \mathbf{y}_{t-k}\right) I\left(s_{t}=j\right)+\varepsilon_{t}
$$

where $\varepsilon_{t} \sim \operatorname{iid}(\mathbf{0}, \boldsymbol{\Sigma})$. The cointegrating vector $\boldsymbol{\beta}=\left(1,-\beta_{U}\right)$, where $-\beta_{U}$ is 
the coefficient of $y_{U, t-1}$. Before fitting (26) to the data, linearity (one regime) is tested against two regimes using the test by Carrasco, Hu and Ploberger (2014) and rejected. It should be noted that in most macroeconomic applications linearity is not tested but the number of regimes is simply assumed known. Testing is important, however, for the reason already mentioned: the MS-AR model, like the TAR and STAR models, is not identified when the true relationship is linear.

It is common to estimate the cointegrating relationship from the linear VAR and keep it fixed in (26). Warne and Vredin (2006) instead construct a grid for $\beta_{U}$ and estimate the other parameters conditionally on values of $\beta_{U}$ in the grid using the EM algorithm. The estimated equations are evaluated using misspecification tests in Hamilton (1996) and their vector generalisations and found adequate. The authors estimate a $95 \%$ confidence interval for $\beta_{U}$ (its estimate equals $\widehat{\beta}_{U}=0.038$ ) by using a grid as explained in the paper. The interval contains zero, and the conclusion is that inflation is actually stationary, whereas there is may be a stochastic trend in unemployment. The estimated regimes are interpreted as low and high inflation ones, and the outcomes pertaining to the original research question are discussed.

When the dimension of the model increases, estimation of VMSAR models often becomes numerically very demanding. In such a situation, Bayesian methods may help. It is not possible to discuss Bayesian VMSAR models in detail here, but a reference is made to the paper by Sims, Waggoner and Zha (2008). The authors show how parameter restrictions in the transition matrix $\mathbf{P}=\left[p_{i j}\right]$, where $p_{i j}$ is defined as in (10) make the VMSAR model a flexible and applicable tool in many situations. For example, it may be used to model structural shifts as well as incremental changes in parameters over time.

Sims et al. (2008) discuss the issue of constructing prior distributions for parameters. They consider the case in which both the mean and the variance of the process are changing over time. They develop a new estimation method that is computationally more efficient than the widely used Monte Carlo EM method. The application in the paper is to the trivariate vector series consisting of the logarithm of the GDP, an inflation variable and the federal funds rate. Nine different models are specified and estimated. The ones with three or four regimes in which only the variance is switching are found to have the best fit, measured by the Marginal Data Density, a concept defined in the paper. 


\subsection{Vector random coefficient autoregressive models}

Assuming coefficients of a linear VAR model to be random generates another family of nonlinear models. Consider the following VAR model

$$
\mathbf{y}_{t}=\sum_{j=1}^{p} \boldsymbol{\Phi}_{j t} \mathbf{y}_{t-j}+\varepsilon_{t}
$$

where $\mathbf{y}_{t}$ is an $m \times 1$ vector, $\boldsymbol{\Phi}_{j t}, j=1, \ldots, p$, are stochastic $m \times m$ parameter matrices, and $\boldsymbol{\varepsilon}_{t} \sim \operatorname{iid}(\mathbf{0}, \boldsymbol{\Sigma})$. Define the $m \times p m$ matrix $\boldsymbol{\Phi}_{t}=$ $\left(\boldsymbol{\Phi}_{1 t}, \ldots, \boldsymbol{\Phi}_{p t}\right)$ and vectorise it into a $p m^{2}$-vector $\boldsymbol{\phi}_{t}=\operatorname{vec}\left(\boldsymbol{\Phi}_{t}\right)$. Nicholls and Quinn $(1981 \mathrm{a}, \mathrm{b})$ assumed that $\phi_{t} \sim \operatorname{iid}(\phi, \Omega)$ and considered stationarity conditions and asymptotic properties of least squares estimators of parameters of this vector random coefficient autoregressive (VRCAR) model. More recently, as in the univariate case, in economic applications the focus has been on (27) such that $\phi_{t}=\phi_{t-1}+\boldsymbol{\nu}_{t}$ with $\boldsymbol{\nu}_{t} \sim \operatorname{iid} \mathcal{N}(\mathbf{0}, \boldsymbol{\Omega})$. Furthermore, $\operatorname{cov}\left(\varepsilon_{t}, \boldsymbol{\nu}_{t}\right)=\boldsymbol{\Lambda}$. This means that the sequence $\left\{\phi_{t}\right\}$, instead of being iid, is a random walk without drift. The paths of the individual coefficients may diverge and make $\left\{\mathbf{y}_{t}\right\}$ an explosive sequence.

Cogley and Sargent (2001) apply this VRCAR model to studying the relationship between inflation, unemployment and the real interest rate. The approach is Bayesian, and the values of $\phi_{t}$ are obtained by simulation after postulating prior distributions for the starting-value $\phi_{0}$ and the hyperparameters $\boldsymbol{\Sigma}, \boldsymbol{\Omega}$ and $\boldsymbol{\Lambda}$. However, since the variance of inflation in this model approaches infinity over time, which, as the authors write, 'cannot be optimal for a central bank that minimizes a loss function involving the variance of inflation', in simulations the draws from the conditional distribution of $\phi_{t}$ given $\phi_{t-1}$ and $\Omega$ leading to explosive roots of the lag polynomial of (27) at time $t$ are discarded. In fact, the variance of the other two variables approaches infinity as well. This restriction implies that $\left\{\mathbf{y}_{t}\right\}$ is persistent but stationary with unknown dynamic properties. Primiceri (2005), applying a similar (but not identical) model, does not impose such a restriction, the argument being that the observation period is so short that the coefficients do not have time to explode. Whether or not this happens also depends on the properties of $\Omega$.

The argument for fitting a VRCAR model to this dataset is that the dynamic relationship between the variables in $\mathbf{y}_{t}$ is constantly changing, and a model with random walk parameters is therefore better suited for characterising the relationship than, say, a VAR model with constant parameters. In the present example, the reason for fitting this reduced form (in Primiceri's case structural) model to the data is that its time-varying parameter esti- 
mates are expected to provide information about changes in monetary policy over the years.

In applications of nonlinear models that nest a linear VAR, testing linearity against these models is possible and, as has been discussed, even necessary. There does not seem to exist a test of a linear VAR against the type of parameter change in the VRCAR model of Cogley and Sargent (2001). (There does not seem to exist a test in which the VRCAR model would be the null hypothesis.) The test by Nyblom (1989) comes closest as its alternative is that the parameter vector is a random walk. In Cogley and Sargent (2005) the test is carried out and the null hypothesis is not rejected. Some tests of the linear VAR against a structural break yield the same result. The authors argue that these tests have low power against their VRCAR. Their conclusion is that 'a failure to reject should not be construed as an embarrassment to time-varying parameter models'. A practical conclusion would be that since the null hypothesis is not rejected, the linear VAR ought to be preferred to the computationally more complicated nonlinear VRCAR model.

The VRCAR models of Cogley and Sargent (2005) and Primiceri (2005) also contain a time-varying error covariance matrix, based on stochastic volatility. Since the focus in this chapter is on the conditional mean models and because of space restrictions, this extension is not considered here. The aforementioned tests, however, are performed under the assumption that the error covariance matrix is constant over time.

\section{$5 \quad$ Nonlinear panel models}

Although this article has concentrated on pure time series models, there is an important related area worth mentioning, namely the nonlinear panel models. In these models the time dimension that contains nonlinearity is completed with cross sections. There exist two popular nonlinear panel models: the panel threshold regression (PTR) model by Hansen (1999) and the panel smooth transition regression (PSTR) model by González, Teräsvirta and van Dijk (2005). In the multi-threshold form the PTR model has the following representation:

$$
y_{i t}=\mu_{i}+\sum_{j=1}^{r} \phi_{j}^{\prime} \mathbf{x}_{i t} I\left(c_{j-1}<s_{i t} \leq c_{j}\right)+\varepsilon_{i t}
$$

$i=1, \ldots, N$, where $y_{i t}$ is a scalar, $\mathbf{x}_{i t}$ is a vector of regressors for the crosssectional unit $i$, the nonzero vectors $\phi_{j} \neq \phi_{k}$ for all $j \neq k, s_{i t}$ is the threshold variable for this unit, and the error term $\varepsilon_{i t} \sim \operatorname{iid}\left(0, \sigma^{2}\right)$. The parameters 
$c_{0}=-\infty$ and $c_{r}=\infty$ as in (4). Hansen (1999) considers linearity testing, determining $r$, and estimation by nonlinear least squares.

The PSTR model is similar to (28) and is defined by the following equation:

$$
y_{i t}=\mu_{i}+\lambda_{t}+\phi_{0}^{\prime} \mathbf{x}_{i t}+\sum_{j=1}^{r} \phi_{j}^{\prime} \mathbf{x}_{i t} G_{j}\left(s_{i t}^{(j)}\right)+\varepsilon_{i t}
$$

where $\lambda_{t}$ is a time-specific variable, for example a trend, common to all units $i$, and $\varepsilon_{i t} \sim \operatorname{iid}\left(0, \sigma_{i}^{2}\right), i=1, \ldots, N$, that is, heteroskedasticity is allowed in cross sections. The transition function equals

$$
G_{j}\left(s_{i t}^{(j)}\right)=\left(1+\exp \left\{-\gamma_{j} \prod_{k=1}^{K}\left(s_{i t}^{(j)}-c_{j}\right)\right\}\right)^{-1}
$$

where different transitions can have different transition variables for each $i$ and, typically, $K=1$ or $K=2$. Modelling problems include determining $r$ and $K$, and they are discussed in the paper.

Both models have been applied to macroeconomic problems and datasets. For example, the relationship between economic growth and inflation ('the growth-inflation nexus') can be perceived as a nonlinear phenomenon. Applications of nonlinear panel models to studying it include Espinoza, Leon and Prasad (2011), Omay and Öznur Kan (2010) and Seleteng, Bittencourt and van Eyden (2013). The Feldstein-Horioka puzzle of positive savinginvestment correlations may be regarded as another one. Fouquau, Hurlin and Rabaud (2008) considered the relationship between these two variables for 24 OECD countries annually from 1960 to 2000 using the PSTR model.

\section{Final remarks and suggestions for further reading}

Due to the large number of nonlinear models applied to macroeconomic problems, many such models have not received the attention they might deserve. For more information, the reader has to turn to other sources. For an overview specifically focussing on macroeconometric time series, see Granger (2001). For an authoritative treatment of univariate TAR models the reader is referred to Tong (1990). Hansen (2011) contains a comprehensive account of economic applications of these models. Teräsvirta, Tjøstheim and Granger (2010) take up a number of nonlinear models not discussed in this work, including nonparametric nonlinear models. Among other things, they also discuss building STAR and TAR models. Nonparametric models 
play a central role in Fan and Yao (2003). Univariate nonlinear models are surveyed in Teräsvirta (2006b), and STAR models in particular in van Dijk, Teräsvirta and Franses (2002). The volume of Krolzig (1997) on Markovswitching models has already been mentioned. There exists a rather recent survey of vector TAR and STAR models by Hubrich and Teräsvirta (2013).

Since space is limited, forecasting nonlinear (macro)economic time series has not been considered either. For two examples that involve macroeconomic variables, see Stock and Watson (1999) and Teräsvirta, van Dijk and Medeiros (2005). Teräsvirta (2006a) is a general survey on forecasting with nonlinear models.

\section{References}

Andel, J.: 1976, Autoregressive series with random parameters, Mathematische Operationsforschung und Statistik 5, 735-741.

Anderson, H. M.: 1997, Transaction costs and non-linear adjustment towards equilibrium in the US treasury bill market, Oxford Bulletin of Economics and Statistics 59, 465-484.

Bacon, D. W. and Watts, D. G.: 1971, Estimating the transition between two intersecting straight lines, Biometrika 58, 525-534.

Baker, S. R., Bloom, N. and Davis, S. J.: 2016, Measuring economic policy uncertainty, Quarterly Journal of Economics 131, 1593-1636.

Balke, N. S. and Fomby, T. B.: 1997, Threshold cointegration, International Economic Review 38, 627-645.

Bauwens, L., Lubrano, M. and Richard, J.-F.: 1999, Bayesian Inference in Dynamic Econometric Models, Oxford University Press, Oxford.

Bec, F. and Rahbek, A.: 2004, Vector equilibrium correction models with non-linear discontinuous adjustments, Econometrics Journal 7, 628-651.

Caggiano, G., Castelnuovo, E. and Figueres, J. M.: 2017, Economic policy uncertainty and unemployment in the United States: A nonlinear approach, Economics Letters 151, 31-34.

Caggiano, G., Castelnuovo, E. and Groshenny, N.: 2014, Uncertainty shocks and unemployment dynamics in U.S. recessions, Journal of Monetary Economics 67, 78-92. 
Cai, B., Gao, J. and Tjøstheim, D.: 2017, A new class of bivariate threshold cointegration models, Journal of Business 85 Economic Statistics 35, 288-305.

Camacho, M.: 2004, Vector smooth transition regression models for US GDP and the composite index of leading indicators, Journal of Forecasting 23, 173-196.

Carrasco, M., Hu, L. and Ploberger, W.: 2014, Optimal test for markov switching parameters, Econometrica 82, 765-784.

Chan, K. S. and Tong, H.: 1986, On estimating thresholds in autoregressive models, Journal of Time Series Analysis 7, 178-190.

Cogley, T. and Sargent, T. J.: 2001, Evolving post-World War II U.S. inflation dynamics, NBER Macroeconomics Annual 16, 331-373.

Cogley, T. and Sargent, T. J.: 2005, Drifts and volatilities: monetary policies and outcomes in the post WWII US, Review of Economic Dynamics 8, 262-302.

Douc, R., Moulines, E. and Rydén, T.: 2004, Asymptotic properties of the maximum likelihood estimator in autoregressive models with Markov regime, Annals of Statistics 32, 2254-2304.

Engle, R. F.: 1982, Autoregressive conditional heteroscedasticity with estimates of the variance of United Kingdom inflation, Econometrica 50, 987-1007.

Ericsson, N. R., Hendry, D. F. and Prestwich, K. M.: 1998, The demand for broad money in the United Kingdom, 1878-1993, Scandinavian Journal of Economics 100, 289-324.

Espinoza, R., Leon, H. and Prasad, A.: 2011, When should we worry about inflation?, World Bank Economic Review 26, 100-127.

Fair, R. C. and Jaffee, D. M.: 1972, Methods of estimation for markets in disequilibrium, Econometrica 40, 497-514.

Fan, J. and Yao, Q.: 2003, Nonlinear Time Series. Nonparametric and Parametric Methods, Springer, New York.

Fouquau, J., Hurlin, C. and Rabaud, I.: 2008, The Feldstein-Horioka puzzle: A panel smooth transition regression approach, Economic Modelling 25, 284-299. 
Galvão, A. B. C.: 2006, Structural break threshold VARs for predicting US recessions using the spread, Journal of Applied Econometrics 21, 463487.

Gefang, D.: 2012, Money-output causality revisited - A Bayesian logistic smooth transition VECM perspective, Oxford Bulletin in Economics and Statistics 74, 131-151.

Goldfeld, S. M. and Quandt, R. E.: 1972, Nonlinear Methods in Econometrics, North-Holland, Amsterdam.

González, A., Teräsvirta, T. and van Dijk, D.: 2005, Panel smooth transition regression models, Working Paper Series in Economics and Finance 604, Stockholm School of Economics, Stockholm.

Granger, C. W. J.: 2001, Overview of nonlinear macroeconometric models, Macroeconomic Dynamics 5, 466-481.

Haggan, V. and Ozaki, T.: 1981, Modelling non-linear random vibrations using an amplitude-dependent autoregressive time series model, Biometrika 68, 189-196.

Hamilton, J. D.: 1989, A new approach to the economic analysis of nonstationary time series and the business cycle, Econometrica 57, 357-384.

Hamilton, J. D.: 1996, Specification testing in Markov-switching time-series models, Journal of Econometrics 70, 127-157.

Hansen, B. E.: 1996, Inference when a nuisance parameter is not identified under the null hypothesis, Econometrica 64, 413-430.

Hansen, B. E.: 1999, Threshold effects in non-dynamic panels: Estimation, testing and inference, Journal of Econometrics 93, 345-368.

Hansen, B. E.: 2011, Threshold autoregression in economics, Statistics and Its Interface 4, 123-127.

Hubrich, K. and Teräsvirta, T.: 2013, Thresholds and smooth transitions in vector autoregressive models, in T. B. Fomby, L. Kilian and A. Murphy (eds), VAR models in macroeconomics - new developments and applications: Essays in honor of Christopher A. Sims, Vol. 32 of Advances in Econometrics, Emerald Group Publishing, Bingley, pp. 273-326. 
Jansen, E. S. and Teräsvirta, T.: 1996, Testing parameter constancy and super exogeneity in econometric equations, Oxford Bulletin in Economics and Statistics 58, 735-763.

Koop, G., Pesaran, M. H. and Potter, S. M.: 1996, Impulse response analysis in nonlinear multivariate models, Journal of Econometrics 74, 119-147.

Koop, G. and Potter, S.: 2000, Nonlinearity, structural breaks, or outliers in economic time series?, in W. A. Barnett, D. F. Hendry, S. Hylleberg, T. Teräsvirta, D. Tjøstheim and A. Würtz (eds), Nonlinear econometric modeling in time series analysis, number 11 in International Symposia in economic theory and econometrics, Cambridge University Press, Cambridge, pp. $61-78$.

Koop, G. and Potter, S. M.: 2001, Are apparent findings of nonlinearity due to structural instability in economic time series?, Econometrics Journal 4, $37-55$.

Krolzig, H.-M.: 1997, Markov-Switching Vector Autoregressions Modelling, Statistical Inference and Applications to Business Cycle Analysis, Springer, Berlin.

Lanne, M. and Saikkonen, P.: 2002, Threshold autoregressions for strongly autocorrelated time series, Journal of Business and Economic Statistics 20, 282-289.

Lindgren, G.: 1978, Markov regime models for mixed distributions and switching regressions, Scandinavian Journal of Statistics 5, 81-91.

Lundbergh, S., Teräsvirta, T. and van Dijk, D.: 2003, Time-varying smooth transition autoregressive models, Journal of Business and Economic Statistics 21, 104-121.

Maddala, G. S.: 1977, Econometrics, McGraw-Hill, New York.

Maddala, G. S.: 1983, Limited-Dependent and Qualitative Variables in Econometrics, Cambridge University Press, Cambridge.

Maddala, G. S. and Nelson, F. D.: 1974, Maximum likelihood methods for models of markets in disequilibrium, Econometrica 42, 1013-1030.

Montgomery, A. R., Zarnowitz, V., Tsay, R. S. and Tiao, G. C.: 1998, Forecasting the U.S. unemployment rate, Journal of the American Statistical Association 93, 478-493. 
Nicholls, D. F. and Quinn, B. G.: 1981a, The estimation of multivariate random coefficient autoregressive models, Journal of Multivariate Analysis 11, 544-555.

Nicholls, D. F. and Quinn, B. G.: 1981b, Multiple autoregressive models with random coefficients, Journal of Multivariate Analysis 11, 185-198.

Nicholls, D. F. and Quinn, B. G.: 1982, Random Coefficient Autoregressive Models: An Introduction, Springer, New York.

Nyblom, J.: 1989, Testing for the constancy of parameters over time, Journal of the American Statistical Association 84, 223-230.

Omay, T. and Öznur Kan, E.: 2010, Re-examining the threshold effects in the inflation-growth nexus with cross-sectionally dependent non-linear panel: Evidence from six industrialized economies, Economic Modelling 27, 996-1005.

Primiceri, G. E.: 2005, Time varying structural vector autoregressions and monetary policy, Review of Economic Studies 72, 821-852.

Quandt, R. E.: 1958, The estimation of parameters of a linear regression system obeying two separate regimes, Journal of the American Statistical Association 53, 873-880.

Saikkonen, P.: 2008, Stability of regime switching error correction models under linear cointegration, Econometric Theory 24, 294-318.

Schleer, F. and Semmler, W.: 2015, Financial sector and output dynamics in the euro area: Non-linearities reconsidered, Journal of Macroeconomics 46, 235-263.

Seleteng, M., Bittencourt, M. and van Eyden, R.: 2013, Non-linearities in inflation-growth nexus in the SADC region: A panel smooth transition regression approach, Economic Modelling 30, 149-156.

Sims, C. A., Waggoner, D. F. and Zha, T.: 2008, Methods for inference in large multple equation Markov-switching models, Journal of Econometrics 146, 255-274.

Stock, J. H. and Watson, M. W.: 1999, A comparison of linear and nonlinear univariate models for forecasting macroeconomic time series, in R. F. Engle and H. White (eds), Cointegration, Causality and Forecasting. A Festschrift in Honour of Clive W.J. Granger, Oxford University Press, Oxford, pp. 1-44. 
Swamy, P. A. V. B. and Tavlas, G. S.: 1995, Random coefficient models: theory and applications, Journal of Economic Surveys 9, 165-196.

Teräsvirta, T.: 1994, Specification, estimation, and evaluation of smooth transition autoregressive models, Journal of the American Statistical Association 89, 208-218.

Teräsvirta, T.: 1998, Modeling economic relationships with smooth transition regressions, in A. Ullah and D. E. Giles (eds), Handbook of Applied Economic Statistics, Dekker, New York, pp. 507-552.

Teräsvirta, T.: 2006a, Forecasting economic variables with nonlinear models, in G. Elliott, C. W. J. Granger and A. Timmermann (eds), Handbook of Economic Forecasting, Vol. 1, Elsevier, Amsterdam, pp. 413-457.

Teräsvirta, T.: 2006b, Univariate nonlinear time series, in T. C. Mills and K. Patterson (eds), Palgrave Handbook of Econometrics: Volume 1, Econometric Theory, Palgrave Macmillan, Basingstoke, pp. 396-424.

Teräsvirta, T. and Anderson, H. M.: 1992, Characterizing nonlinearities in business cycles using smooth transition autoregressive models, Journal of Applied Econometrics 7, S119-S136.

Teräsvirta, T. and Eliasson, A.-C.: 2001, Non-linear error correction and the UK demand for broad money, 1878-1993, Journal of Applied Econometrics 16, 277-288.

Teräsvirta, T., Tjøstheim, D. and Granger, C. W. J.: 2010, Modelling Nonlinear Economic Time Series, Oxford University Press, Oxford.

Teräsvirta, T., van Dijk, D. and Medeiros, M. C.: 2005, Smooth transition autoregressions, neural networks, and linear models in forecasting macroeconomic time series: A re-examination, International Journal of Forecasting 21, 755-774.

Tong, H.: 1990, Non-Linear Time Series. A Dynamical System Approach, Oxford University Press, Oxford.

Tong, H. and Lim, K. S.: 1980, Threshold autoregression, limit cycles and cyclical data, Journal of the Royal Statistical Society B 42, 245-292.

Tsay, R. S.: 1998, Testing and modeling multivariate threshold models, Journal of the American Statistical Association 93, 1188-1202. 
van Dijk, D. and Franses, P. H.: 1999, Modeling multiple regimes in the business cycle, Macroeconomic Dynamics 3, 311-340.

van Dijk, D., Teräsvirta, T. and Franses, P. H.: 2002, Smooth transition autoregressive models - a survey of recent developments, Econometric Reviews 21, 1-47.

Warne, A. and Vredin, A.: 2006, Unemployment and inflation regimes, Studies in Nonlinear Dynamics and Econometrics 10, Issue 2, Article 2. 
2017-15: $\quad$ Andrea Barletta, Paolo Santucci de Magistris and Francesco Violante: A NonStructural Investigation of VIX Risk Neutral Density

2017-16: Davide Delle Monache, Stefano Grassi and Paolo Santucci de Magistris: Does the ARFIMA really shift?

2017-17: Massimo Franchi and Søren Johansen: Improved inference on cointegrating vectors in the presence of a near unit root using adjusted quantiles

2017-18: $\quad$ Matias D. Cattaneo, Michael Jansson and Kenichi Nagasawa: Bootstrap-Based Inference for Cube Root Consistent Estimators

2017-19: Daniel Borup and Martin Thyrsgaard: Statistical tests for equal predictive ability across multiple forecasting methods

2017-20: $\quad$ Tommaso Proietti and Alessandro Giovannelli: A Durbin-Levinson Regularized Estimator of High Dimensional Autocovariance Matrices

2017-21: Jeroen V.K. Rombouts, Lars Stentoft and Francesco Violante: Variance swap payoffs, risk premia and extreme market conditions

2017-22: Jakob Guldbæk Mikkelsen: Testing for time-varying loadings in dynamic factor models

2017-23: $\quad$ Roman Frydman, Søren Johansen, Anders Rahbek and Morten Nyboe Tabor: The Qualitative Expectations Hypothesis: Model Ambiguity, Concistent Representations of Market Forecasts, and Sentiment

2017-24: Giorgio Mirone: Inference from the futures: ranking the noise cancelling accuracy of realized measures

2017-25: $\quad$ Massimiliano Caporin, Gisle J. Natvik, Francesco Ravazzolo and Paolo Santucci de Magistris: The Bank-Sovereign Nexus: Evidence from a nonBailout Episode

2017-26: $\quad$ Mikkel Bennedsen, Asger Lunde and Mikko S. Pakkanen: Decoupling the shortand long-term behavior of stochastic volatility

2017-27: $\quad$ Martin M. Andreasen, Jens H.E. Christensen and Simon Riddell: The TIPS Liquidity Premium

2017-28: Annastiina Silvennoinen and Timo Teräsvirta: Consistency and asymptotic normality of maximum likelihood estimators of a multiplicative time-varying smooth transition correlation GARCH model

2017-29: Cristina Amado, Annastiina Silvennoinen and Timo Teräsvirta: Modelling and forecasting WIG20 daily returns

2017-30: Kim Christensen, Ulrich Hounyo and Mark Podolskij: Is the diurnal pattern sufficient to explain the intraday variation in volatility? A nonparametric assessment

2017-31: $\quad$ Martin M. Andreasen, Jens H.E. Christensen and Glenn D. Rudebusch: Term Structure Analysis with Big Data

2017-32: Timo Teräsvirta: Nonlinear models in macroeconometrics 